\title{
Time-Varying Parameter Estimation under Stochastic Perturbations Using LSM
}

Jesica Escobar

Division of Automatic Control

E-mail: escobar@isy.liu.se

13th June 2011

Report no.: LiTH-ISY-R-3019

Submitted to IMA Journal of Mathematical Control and Information

Address:

Department of Electrical Engineering

Linköpings universitet

SE-581 83 Linköping, Sweden

WWW: http://WwW. control.isy.liu.se

AUTOMATIC CONTROL
REGLERTEKNIK
LINKÖPINGS UNIVERSITET

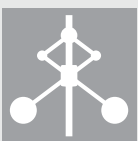

Technical reports from the Automatic Control group in Linköping are available from http://www. control.isy.liu.se/publications. 


\begin{abstract}
In this paper, we deal with the problem of continuous-time time-varying parameter estimation in stochastic systems, under 3 different kinds of stochastic perturbations: additive and multiplicative white noise, and colored noise. The proposed algorithm is based on the Least Squares Method with forgetting factor. Some numerical examples illustrate the effectiveness of the proposed algorithm. An analysis of the estimation error for the system under the 3 different kinds of perturbations is presented.
\end{abstract}

Keywords: Stochastic systems, parameter estimation, least squares method. 


\title{
TIME-VARYING PARAMETER ESTIMATION UNDER STOCHASTIC PERTURBATIONS USING LSM
}

\author{
JESICA ESCOBAR
}

\begin{abstract}
In this paper, we deal with the problem of continuous-time timevarying parameter estimation in stochastic systems, under 3 different kinds of stochastic perturbations: additive and multiplicative white noise, and colored noise. The proposed algorithm is based on the Least Squares Method with forgetting factor. Some numerical examples illustrate the effectiveness of the proposed algorithm. An analysis of the estimation error for the system under the 3 different kinds of perturbations is presented.
\end{abstract}

\section{INTRODUCTION}

Stochastic control theory deals with dynamic systems, described by difference or differential equations, and subject to disturbances which are characterized as stochastic processes [1]. Some examples would be the noise in electronic equipment, the random fluctuations of various quantities in a process control system, and the movement of prices on the Stock Exchange [2]. None of these cases can be represented realistically by a completely deterministic mathematical model, since this would imply, for example, the ability to predict exactly their future evolution. Thus we are forced back to probabilistic modeling which will enable us to say at least how the processes will behave in some average sense. The tools required to solve all these problems are fairly recent developments.

The filtering and prediction theory developed by Wiener and Kolmogorov is one of the cornerstones in stochastic control theory. This theory makes it possible to extract a signal from observations of signal and disturbances.

Historically, system identification has been motivated by the need for designing better control systems. It can be seen as the interface between the real world of applications and the mathematical world of control theory and model abstractions [3] . Basically, the class of linear and nonlinear systems whose equations are linear in the parameters was assumed to be of a stochastic nature (see [4],[5],[6]). Identification procedures in continuous time often deal with the so called "white noise", where the standard Wiener process participates in the representation of a stochastic dynamic description [7], [8].

Date: June, 2011.

Key words and phrases. Stochastic systems, parameter estimation, least squares method.

Division of Automatic Control, University of Linkoping, 58183 LINKOPING Sweden.

E-mail: escobar@isy.liu.se.

I want to thank to the Mexican Council of Science and Technology (CONACYT) for the support given to this project.

This paper is in final form and no version of it will be submitted for publication elsewhere. 
In this topic many techniques have been developed; system identification in discrete time of constant parameters is usually addressed by the least squares method (LSM) (see [9] and [10]). This method has been very successful and it is very popular in applications. The Approximate Maximum Likelihood Estimation (AMLE) is an algorithm for estimating the states and parameters of models described by stochastic differential equations (see [11], [12]). This method presents a better performance compared with a Kalman filter based maximum likelihood method, and has been used in cases in which some states are unmeasured. For continuous time system in [13] a continuous-time autoregressive method (CAR) is proposed, the additive measurement noise is modeled as a continuous time white noise, and then the direct Leas Squares method and average sampling are applied. In some cases, the advantage of a one-dimensional observation, is used to obtain an easy way to compute the exact likelihood using the Kalman filter recursion, which allows to implement an easy numerical maximization of the likelihood (see [14]). Instrumental variable (IV) methods using filtered inputs and delayed filtered outputs as IV are also used in continuous-time systems identified through an approximated discrete-time estimation model [15].

Some adaptive observers are proposed in [16] for the purpose of recursive join estimation of state and parameters in continuous-time state space systems; this algorithm makes use of a time varying gain matrix with a forgetting factor for parameter estimation. Some other adaptive techniques, like extended least squares identification (ELS), are presented in [19], here a weighting is introduced into the ELS scheme according to a stability measure. For time-varying system, the performance of the standard forgetting factor recursive least squares (RLS) algorithms when used for tracking time-varying linear regression models, and the forgetting factor least squares (FFLS) algorithm in the stochastic framework have been analyzed in [17] and [18].

For perturbations that are colored or correlated, the spectral analysis can be used for the parameter estimation [20]. In such cases of interest to express a spectral density as a sum of two terms: the first term corresponds to the undisturbed signal while the other refers to the measurement noise, then the signal and the measurement noise are treated as ARMA processes. The LSM with forgetting factor is suggested too for time-varying matrix estimation in stochastic continuoustime model under colored noise .

In this paper we deal with the problem of time-varying parameter estimation in continuous time with, additive white noise, multiplicative white noise, and colored noise. The proposed algorithm is based on the LSM with forgetting factor. The main idea is to compare the effectiveness of the LSM in systems with this kind of stochastic perturbations.

Main contribution.

- the proposed method does not apply any kind of preliminary discretization of the process.

- it is not used any pre-filtering or whitening for the colored noise.

- the dependence on the degree of color is presented, as well as the analysis of the convergence zone.

- the method can be applied under different kinds of stochastic perturbations.

Main restrictions.

- the rate of the parameter variation is assumed to be bounded. 
- the 8-th moments of the colored noise.

Paper Structure. In the second section it is presented the problem formulation, the estimation algorithms for the 3 cases are explained in the third section. Then the analysis of the estimation error is presented. Finally a numerical example presents the performance of the algorithm.

\section{Problem formulation}

Consider the stochastic continuous-time system given by the following differential equations

$$
\begin{gathered}
d x_{t}=A_{t} \zeta\left(x_{t}\right) d t+f_{t} d t+B_{t} d s_{t} \\
d s_{t}=H_{t} s_{t} d t+\sigma_{t} d W_{t} \\
\forall t \in[0, T](T<\infty) \text { with } x(0, \omega)=x_{0}(\omega)
\end{gathered}
$$

where $\left(\Omega, \mathcal{F},\left\{\mathcal{F}_{t}\right\}_{t \geq 0}, P\right)$ is the given filtered probability space where a standard vector-valued motion $\left(W_{t} \in \mathbb{R}^{l}, t \geq 0\right.$ ) (with $W_{0}=0$ ) is defined. $\left\{\mathcal{F}_{t}\right\}_{t \geq 0}$ is assumed to be the natural filtration generated by $\left(W_{t}, t \geq 0\right)$ and augmented by the $P$-null sets from $\mathcal{F}$.

In this system $A_{t}$ is the time-varying matrix to be identified, $f_{t}$ is a bounded excitation input, $\zeta: \mathbb{R}^{n} \rightarrow \mathbb{R}^{m}$ is a known (measurable) nonlinear Lipschitz vector function or, a "regressor", and $\sigma_{t}$ is bounded too. For the noise $B_{t} d s_{t}$ we will consider 3 different cases:

1. $B_{t}=I$ and $d s_{t}=\sigma_{t} d W_{t}$, i.e. when the noise is a standard, additive white noise.

2. $B_{t}=I$ and $d s_{t}=\sigma_{t} x_{t} d W_{t}$, i.e. a multiplicative white noise.

3. $B_{t} \in \mathbb{R}^{n \times m}$ and $d s_{t}=H_{t} s_{t} d t+\sigma_{t} d W_{t}$, i.e. a colored noise.

In all the cases $W_{t}$ is a standard vector Wiener process, i.e.,

$$
E\left\{W_{t} \mid \mathcal{F}_{s<t}\right\} \stackrel{a . s .}{=} 0, E\left\{W_{t} W_{s}^{\top} \mid \mathcal{F}_{r<(s \wedge t)}\right\} \stackrel{a . s .}{=} \delta_{t-s} I_{l \times l}
$$

The main problem is to design the estimate $\hat{A}_{t}$ based only on the available data, up to time $t$. Here we will estimate the upper error bound $\theta$

$$
\varlimsup_{t \rightarrow \infty} E\left\{\operatorname{tr}\left[\left(A_{t}-\hat{A}_{t}\right)^{\top} \tilde{\Gamma}_{t}^{-2}\left(A_{t}-\hat{A}_{t}\right)\right]\right\} \leq \theta
$$

for the matrix LSM-procedure with a scalar forgetting factor for the 3 cases.

The following assumptions will be necessary for this article:

A1. For all $x$, the regressor $\zeta(x)$ is quasi-linear, i.e., it satisfies the global Lipschitz condition

$$
\|\zeta(x)\| \leq L\|x\|
$$

A2. The parametric matrix $A_{t}$ is differentiable and $\dot{A}_{t}$ is bounded for all $t \geq 0$

$$
\left\|\dot{A}_{t}\right\| \leq \delta_{A}
$$

A3. The noise power $\sigma_{t}$ is bounded, i.e., for any $t \geq 0$

$$
\operatorname{tr}\left\{\sigma_{t} \sigma_{t}^{\top}\right\} \leq D_{\sigma}<\infty
$$

this is required in order to guarantee the existence of any moment more than 2 . 
A4. The plant is considered to be $L_{8}$-stable, i.e., there exist the bounded 8-th moments

$$
\begin{aligned}
& \sup _{t>0} E\left\{\left\|x_{t}\right\|^{8}\right\} \leq X_{8}^{+}<\infty \\
& \sup _{t>0} E\left\{\left\|s_{t}\right\|^{8}\right\} \leq S_{8}^{+}<\infty
\end{aligned}
$$

that do not depend of initial condition.

A5. The matrix norm of variations $\Delta H_{t}$ and $B_{t}$, for all $t \geq 0$, are bounded

$$
\left\|\Delta H_{t}\right\| \leq \Delta^{+}, \operatorname{tr}\left\{B_{t} B_{t}^{\top}\right\} \leq D_{B}<\infty
$$

these assumptions are required to guarantee the stability of the model.

Remark 1. Here we deal with the current regressor's covariance

$$
\tilde{\Gamma}_{t}^{-1}={ }_{0}^{t} \zeta\left(x_{\tau}\right) \zeta^{\top}\left(x_{\tau}\right) d \tau
$$

and its various combinations, in particular the Cauchy-Schwartz inequality

$$
\begin{gathered}
E\left\{\operatorname{tr}\left[\left(A_{t}-\hat{A}_{t}\right)^{\top} \tilde{\Gamma}_{t}^{-2}\left(A_{t}-\hat{A}_{t}\right)\right]\right\} \leq E\left\{\left\|A_{t}-\hat{A}_{t}\right\|^{2}\left\|\tilde{\Gamma}_{t}^{-1}\right\|^{2}\right\} \leq \\
\sqrt{E\left\{\left\|A_{t}-\hat{A}_{t}\right\|^{4}\right\}} \sqrt{\frac{E\left\{\left\|\tilde{\Gamma}_{t}^{-1}\right\|^{4}\right\}}{\|A\| \sqrt{E\left\{\left\|A_{t}-\hat{A}_{t}\right\|^{4}\right\}}} \sqrt{{ }_{0}^{t} E\left\|\zeta\left(x_{\tau}\right)\right\|^{8} d \tau}} \\
\| A \sqrt{\lambda_{\max }\left(A^{\top} A\right)}
\end{gathered}
$$

assumption $A_{4}$ provides the $L_{8}$-stability that it is required to guarantee, at least, the existence of the left-hand side for all $t \geq 0$.

Remark 2. It is well-known (see [4]) that in stochastic systems it is impossible to estimate time-varying parameters exactly: there always exists a bound of the corresponding accuracy depending on the rate of change of the estimated parameters (even in the "white-noise"case) as well as on the noise power. Here we use the "unusual" measure of the estimation quality, namely, $E\left\{\operatorname{tr}\left(\Delta A_{t}^{\top} \tilde{\Gamma}_{t}^{-2} \Delta A_{t}\right)\right\}$ that under regular "persistent excitation conditions" and when parameters are constant in time (when the forgetting factor $r=1$ and, hence, $\frac{1}{t} \tilde{\Gamma}_{t}^{-1} \underset{t \rightarrow \infty}{\stackrel{a . s .}{\rightarrow}} \tilde{\Gamma}=$ Const) is asymptotically equal to the following one

$$
E\left\{\operatorname{tr}\left(\Delta A_{t}^{\top} \tilde{\Gamma}_{t}^{-2} \Delta A_{t}\right)\right\} \sim t^{2} E\left\{\operatorname{tr}\left(\Delta A_{t}^{\top} \tilde{\Gamma} \Delta A_{t}\right)\right\}
$$

It is not difficult to show that the upper estimate of this "quality function" is proportional to ttr $\Xi$ (where $\Xi$ in fact is the covariance matrix of the regressor). This implies the estimate

$$
E\left\{\operatorname{tr}\left(\Delta A_{t}^{\top} \tilde{\Gamma} \Delta A_{t}\right)\right\} \sim t^{-1} \operatorname{tr} \Xi
$$

and, therefore, $\operatorname{tr} \Xi$ can be treated as the "convergence rate " corresponding to the order of convergence $t^{-1}$. When parameters to be estimated are time-varying the standard LSM (with $r=1$ ) does not work, and it should satisfy $0<r<1$. In this case one has

$$
\sup _{t \geq t_{0}}\left\|\tilde{\Gamma}_{t}^{-1}\right\| \stackrel{a . s}{<} \text { Const }
$$

and the "quality of estimation" may be bounded by a constant $\theta$ (but not by $t \cdot \theta$ ) only. It never can be done as small as we wish, but nevertheless, it can be treated as a "bound for the convergence zone". 
Remark 3. In the white noise case, additive and multiplicative, the system fulfills the existence condition (see [23]) and is pathwise unique. For a system with colored noise, where this is similar to an Ornstein-Uhlenbeck equation, there are some analytical results and some numerical solutions of the differential equations with colored noise (see for example [25]).

\section{Algorithm for Matrix Estimation}

3.1. Additive white noise. First, we will deal with the simplest case, additive white noise. Let's define the system

$$
d x_{t}=\left(A_{t} x_{t}+f_{t}\right) d t+\sigma_{t} d W_{t}
$$

now, back integrating the equation, this can be expressed as follows

$$
x_{t}-x_{t-h}-\int_{t-h}^{t} f_{s^{\prime}} d s^{\prime}=\int_{t-h}^{t} A_{s^{\prime}} x_{s^{\prime}} d s^{\prime}+\int_{t-h}^{t} \sigma_{s^{\prime}} d W_{s^{\prime}}
$$

or, in a regressive form

$$
F_{t, t-h}=A_{t} X_{t, t-h}+\xi_{t, t-h}
$$

where each term can be defined in the following way

$$
\begin{gathered}
F_{t, t-h}:=x_{t}-x_{t-h}-\int_{t-h}^{t} f_{s^{\prime}} d s^{\prime} \\
X_{t, t-h}:=\int_{s^{\prime}=t-h}^{t} x_{s^{\prime}} d s^{\prime} \\
\xi_{t, t-h}:=\int_{t-h}^{t} \sigma_{s^{\prime}} d W_{s^{\prime}}+\int_{t-h}^{t}\left(A_{s^{\prime}}-A_{t}\right) x_{s^{\prime}} d s^{\prime}
\end{gathered}
$$

here $h>0$ is the back step, and $X_{t, t-h}$ defined for $\tau \geq h$. Here the "extended output" $F_{t, t-h}$ and the "extended regressor" $X_{t, t-h}$ are known at each $t \geq 0$. Multiplying $X_{t, t-h}^{\top}$ in the right hand side, and integrating back from $t-h$ to $t$, we get

$$
\begin{gathered}
\int_{t-h}^{t} F_{\tau, \tau-h} X_{\tau, \tau-h}^{\top} d \tau=\int_{t-h}^{t} A_{\tau} X_{\tau, \tau-h} X_{\tau, \tau-h}^{\top} d \tau+\int_{t-h}^{t} \xi_{\tau, \tau-h} X_{\tau, \tau-h}^{\top} d \tau= \\
A_{t} \int_{t-h}^{t} X_{\tau, \tau-h} X_{\tau, \tau-h}^{\top} d \tau+\bar{\xi}_{t, t-h}
\end{gathered}
$$

Now the error extended vector can be defined as follows

$$
\bar{\xi}_{t, t-h}=\int_{t-h}^{t}\left(\left[A_{\tau}-A_{t}\right] X_{\tau, \tau-h}+\xi_{\tau, \tau-h}\right) X_{\tau, \tau-h}^{\top} d \tau
$$


Since the rate of change of the parameters $\left\|A_{s^{\prime}}-A_{t}\right\| \forall s^{\prime} \in[t-h, t]$ is small enough ( $h$ can adopt small values), the estimated matrix $\hat{A}_{t}$ can be defined as the matrix that satisfies the next equality

$$
\begin{gathered}
\int_{t-h}^{t} F_{\tau, \tau-h} X_{\tau, \tau-h}^{\top} d \tau=\hat{A}_{t} \int_{t-h}^{t} X_{\tau, \tau-h} X_{\tau, \tau-h}^{\top} d \tau \\
\left(\hat{A}_{t}\right)_{i, j}=0 \text { for } j \neq m+i, j>m
\end{gathered}
$$

The extended error vector $\bar{\xi}_{t, t-h}$ will represent the estimation error corresponding to the estimated matrix $\hat{A}_{t}$ that satisfies (3.6). If the persistent excitation condition is fulfilled, i.e., if $\forall t \geq h>0$, then

$$
\int_{t-h}^{t} X_{\tau, \tau-h} X_{\tau, \tau-h}^{\top} d \tau>0
$$

and the estimated matrix $\hat{A}_{t}$ can be represented as follows

$$
\begin{aligned}
& \hat{A}_{t}:=\left[\int_{t-h}^{t} F_{\tau, \tau-h} X_{\tau, \tau-h}^{\top} d \tau\right] \Gamma_{t} \\
& \Gamma_{t}:=\left[\int_{t-h}^{t} X_{\tau, \tau-h} X_{\tau, \tau-h}^{\top} d \tau\right]^{-1} \\
& \left(\hat{A}_{t}\right)_{i, j}=0 \text { for } j \neq m+i, j>m
\end{aligned}
$$

or in the alternative way

$$
\begin{aligned}
\hat{A}_{t}= & {\left[\int_{0}^{t} F_{\tau, \tau-h} X_{\tau, \tau-h}^{\top} \chi(\tau \geq t-h) d \tau\right] \Gamma_{t} } \\
\Gamma_{t}:= & {\left[\int_{0}^{t} X_{\tau, \tau-h} X_{\tau, \tau-h}^{\top} \chi(\tau \geq t-h) d \tau\right] } \\
& \left(\hat{A}_{t}\right)_{i, j}=0 \text { for } j \neq m+i, j>m
\end{aligned}
$$

here, $\chi(\tau \geq t-h)$ is the characteristic function defined by

$$
\chi(\tau \geq t-h):=\left\{\begin{array}{lll}
1 & \text { if } \quad \tau \geq t-h \\
0 & \text { if } \tau<t-h
\end{array}\right.
$$

Since this function characterizes the "window" $[t-h, t]$ in the integrals (3.9). Now, instead of $\chi(\tau \geq t-h)$, a different class of "window" can be used, for example, an "extended window" corresponding to the "forgetting factor" that provides the following estimation

$$
\begin{gathered}
\hat{A}_{t}=Y_{t} \Gamma_{t}, t \geq h \\
\left(\hat{A}_{t}\right)_{i, j}=0 \text { for } j \neq m+i, j>m
\end{gathered}
$$


with

$$
\begin{aligned}
Y_{t} & =\int_{0}^{t} F_{\tau, \tau-h} X_{\tau, \tau-h}^{\top} r^{t-\tau} d \tau \\
\Gamma_{t}^{-1}: & \int_{0}^{t} X_{\tau, \tau-h} X_{\tau, \tau-h}^{\top} r^{t-\tau} d \tau
\end{aligned}
$$

where $r$ is the scalar forgetting factor $0<r<1$.

3.1.1. Differential Form of the Estimation Algorithm. The direct derivation of (3.11) and (3.12) yields

$$
\begin{gathered}
\frac{d}{d t} \hat{A}_{t}=Y_{t} \dot{\Gamma}_{t}+\dot{Y}_{t} \Gamma_{t} \\
\left(\hat{A}_{t}\right)_{i, j}=0 \text { for } j \neq m+i, j>m
\end{gathered}
$$

where

$$
\dot{Y}_{t}=F_{\tau, \tau-h} X_{\tau, \tau-h}^{\top}+\int_{0}^{t} F_{\tau, \tau-h} X_{\tau, \tau-h}^{\top} \frac{d}{d t} r^{t-\tau} d \tau
$$

In this case $\frac{d}{d t} r^{t-\tau}=r^{t-\tau} \ln r$. Then, (3.14) can be rewritten as follows:

$$
\begin{gathered}
\dot{Y}_{t}=F_{\tau, \tau-h} X_{\tau, \tau-h}^{\top}+\int_{0}^{t} F_{\tau, \tau-h} X_{\tau, \tau-h}^{\top} \frac{d}{d t} r^{t-\tau} d \tau \\
=F_{\tau, \tau-h} X_{\tau, \tau-h}^{\top}+Y_{t} \ln r
\end{gathered}
$$

In order to calculate $\dot{\Gamma}_{t}$, the identity $\Gamma_{t} \Gamma_{t}^{-1}=I$ will be derived, then we get the following equations

$$
\begin{gathered}
\dot{\Gamma}_{t} \Gamma_{t}^{-1}+\Gamma_{t} \dot{\Gamma}_{t}^{-1}=0 \\
\dot{\Gamma}_{t}=-\Gamma_{t} \dot{\Gamma}_{t}^{-1} \Gamma_{t}
\end{gathered}
$$

the direct derivation of (3.12) yields

$$
\begin{gathered}
\dot{\Gamma}_{t}^{-1}=X_{t, t-h} X_{t, t-h}^{\top}+\int_{0}^{t} X_{\tau, \tau-h} X_{\tau, \tau-h}^{\top} \frac{d}{d t} r^{t-\tau} d \tau= \\
=X_{t, t-h} X_{t, t-h}^{\top}+\Gamma_{t}^{-1} \ln r
\end{gathered}
$$

Replacing $\dot{\Gamma}_{t}^{-1}(3.17)$ in (3.16) we get

$$
\dot{\Gamma}_{t}=-\Gamma_{t} X_{t, t-h} X_{t, t-h}^{\top} \Gamma_{t}-\ln r \Gamma_{t}
$$

Now, replacing (3.15) and (3.18) in (3.13)

$$
\begin{aligned}
\frac{d}{d t} \hat{A}_{t}= & -\hat{A}_{t} X_{t, t-h} X_{t, t-h}^{\top} \Gamma_{t}+F_{t, t-h} X_{t, t-h}^{\top} \Gamma_{t} \\
& =\left(-\hat{A}_{t} X_{t, t-h}+F_{t, t-h}\right) X_{t, t-h}^{\top} \Gamma_{t}
\end{aligned}
$$


Finally, equations (3.18) and (3.19) will form the estimation algorithm:

$$
\begin{gathered}
\frac{d}{d t} \hat{A}_{t}=\left(-\hat{A}_{t} X_{t, t-h}+F_{t, t-h}\right) X_{t, t-h}^{\top} \Gamma_{t} \\
\left(\hat{A}_{t}\right)_{i, j}=0 \text { for } j \neq m+i, j>m \\
\dot{\Gamma}_{t}=-\Gamma_{t} X_{t, t-h} X_{t, t-h}^{\top} \Gamma_{t}-(\ln r) \Gamma_{t} \\
t \geq t_{0}:= \\
\inf _{t}\left\{t \geq 0: \operatorname{det} \Gamma_{t}^{-1}=\operatorname{det}\left(\int_{0}^{t} X_{\tau, \tau-h} X_{\tau, \tau-h}^{\top} r^{t-\tau} d \tau\right)>0\right\} \\
\Gamma_{t_{0}}=\left[\int_{0}^{t_{0}} X_{\tau, \tau-h} X_{\tau, \tau-h}^{\top} r^{t-\tau} d \tau\right]^{-1}, \hat{A}_{t_{0}}=Y_{t_{0}} \Gamma_{t_{0}}
\end{gathered}
$$

In fact, $t_{0}$ is any time just after the moment when the matrix $\Gamma_{t}^{-1}$ is non-singular.

3.2. Multiplicative white noise. For this case, let's define the following system

$$
d x_{t}=\left(A_{t} x_{t}+f_{t}\right) d t+\sigma_{t} x_{t} d W_{t}
$$

first, back integrating (3.21) we get

$$
x_{t}-x_{t-h}-\int_{t-h}^{t} f_{s^{\prime}} d s^{\prime}=\int_{t-h}^{t} A_{s^{\prime}} x_{s^{\prime}} d s^{\prime}+\int_{t-h}^{t} \sigma_{s^{\prime}} x_{s^{\prime}} d W_{s^{\prime}}
$$

or in the regressive form

$$
F_{t, t-h}=A_{t} X_{t, t-h}+\xi_{t, t-h}
$$

where

$$
\begin{gathered}
F_{t, t-h}:=x_{t}-x_{t-h}-\int_{t-h}^{t} f_{s^{\prime}} d s^{\prime} \\
X_{t, t-h}:=\int_{s^{\prime}=t-h}^{t} x_{s^{\prime}} d s^{\prime} \\
\xi_{t, t-h}:=\int_{t-h}^{t} \sigma_{s^{\prime}} x_{s^{\prime}} d W_{s^{\prime}}+\int_{t-h}^{t}\left(A_{s^{\prime}}-A_{t}\right) x_{s^{\prime}} d s^{\prime}
\end{gathered}
$$

is possible to see that the structure is basically the same than in the last case, then is possible to use the same algorithm that was estimated in the previous subsection.

3.3. Colored noise. For this case, consider the continuous time system given by the following differential equations:

$$
\begin{gathered}
d x_{t}=A_{t} \zeta\left(x_{t}\right) d t+f_{t} d t+B_{t} d s_{t} \\
d s_{t}=H_{t} s_{t} d t+\sigma_{t} d W_{t}
\end{gathered}
$$


here, $H_{t}:=H_{0}+\Delta H_{t}$ with $H_{0} \in \mathbb{R}^{n \times m}$ known, $\Delta H_{t} \in \mathbb{R}^{n \times m}$ is unknown but bounded, i.e., $\left\|\Delta H_{t}\right\| \leq \Delta^{+}, B_{t} \in \mathbb{R}^{n \times m}$ is a deterministic known matrix. The system now will be represented in the following extended form

$$
\left.\begin{array}{c}
d x_{t}=z_{t} C_{t} d t+f_{t} d t+B_{t} \sigma_{t} d W_{t}+B_{t} \Delta H_{t} s_{t} d t \\
C_{12} \\
\vdots \\
a_{n m} \\
s_{1} \\
\vdots \\
s_{m}
\end{array}\right], z_{t}:=\left[\begin{array}{cccccccccc}
x_{1} & \cdots & x_{m} & 0 & 0 & \cdots & 0 & g_{11} & \cdots & g_{1 m} \\
\vdots & \ddots & \vdots & \ddots & \vdots & \ddots & \vdots & \vdots & \ddots & \vdots \\
0 & \cdots & 0 & 0 & x_{1} & \cdots & x_{m} & g_{n 1} & \cdots & g_{n m}
\end{array}\right]
$$

notice that this system has a different structure, this is because in this system the perturbations are not independent but correlated.

As in the previous case, we integrate backs (3.26) with $t \geq h$ in the following way

$$
x_{t}-x_{t-h}-\int_{t-h}^{t} f_{s^{\prime}} d s^{\prime}=\int_{t-h}^{t} z_{s^{\prime}} C_{s^{\prime}} d s^{\prime}+\int_{t-h}^{t} B_{s^{\prime}} \sigma_{s^{\prime}} d W_{s^{\prime}}+\int_{t-h}^{t} B_{s^{\prime}} \Delta H_{s^{\prime}} s_{s^{\prime}} d s^{\prime}
$$

or, in the so-called regressive form

$$
\begin{gathered}
F_{t, t-h}=Z_{t, t-h} C_{t}+\xi_{t, t-h} \\
F_{t, t-h}:=x_{t}-x_{t-h}-\int_{t-h}^{t} f_{s^{\prime}} d s^{\prime}, Z_{t, t-h}:=\int_{s^{\prime}=t-h}^{t} z_{s^{\prime}} d s^{\prime} \\
\xi_{t, t-h}:=\int_{t-h}^{t} B_{s^{\prime}} \sigma_{s^{\prime}} d W_{s^{\prime}}+\int_{t-h}^{t} z_{s^{\prime}}\left(C_{s^{\prime}}-C_{t}\right) d s^{\prime}+\int_{t-h}^{t} B_{s^{\prime}} \Delta H_{s^{\prime}} s_{s^{\prime}} d s^{\prime}
\end{gathered}
$$

with $h>0$ as the back step. Multiplying by the left hand side by $Z_{t, t-h}^{\top}$, and integrating back from $t-h$ to $t$, this yields

$$
\begin{gathered}
\int_{t-h}^{t} Z_{\tau, \tau-h}^{\top} F_{\tau, \tau-h} d \tau=\int_{t-h}^{t} Z_{\tau, \tau-h}^{\top} Z_{\tau, \tau-h} C_{\tau} d \tau+\int_{t-h}^{t} Z_{\tau, \tau-h}^{\top} \xi_{\tau, \tau-h} d \tau= \\
\left(\int_{t-h}^{t} Z_{\tau, \tau-h}^{\top} Z_{\tau, \tau-h} d \tau\right) C_{t}+\bar{\xi}_{t, t-h}
\end{gathered}
$$

where

$$
\bar{\xi}_{t, t-h}=\int_{t-h}^{t} Z_{\tau, \tau-h}^{\top}\left(Z_{\tau, \tau-h}\left[C_{\tau}-C_{t}\right]+\xi_{\tau, \tau-h}\right) d \tau
$$

following the same method that was used in the previous subsections, we get the following estimation

$$
\hat{C}_{t}=\Gamma_{t} Y_{t}, t \geq h
$$


with

$$
\begin{aligned}
Y_{t} & =\int_{0}^{t} Z_{\tau, \tau-h}^{\top} F_{\tau, \tau-h} r^{t-\tau} d \tau \\
\Gamma_{t}^{-1} & :=\int_{0}^{t} Z_{\tau, \tau-h}^{\top} Z_{\tau, \tau-h} r^{t-\tau} d \tau
\end{aligned}
$$

3.3.1. Differential form of the estimation algorithm. The direct differentiation of (3.30)and (3.31) implies

$$
\frac{d}{d t} \hat{C}_{t}=\Gamma_{t} \dot{Y}_{t}+\dot{\Gamma}_{t} Y_{t}
$$

where

$$
\begin{gathered}
\dot{Y}_{t}=Z_{\tau, \tau-h}^{\top} F_{\tau, \tau-h}+\int_{0}^{t} Z_{\tau, \tau-h}^{\top} F_{\tau, \tau-h} \frac{d}{d t} r^{t-\tau} d \tau \\
=Z_{\tau, \tau-h}^{\top} F_{\tau, \tau-h}+Y_{t} \ln r
\end{gathered}
$$

and

$$
\begin{gathered}
\dot{\Gamma}_{t}^{-1}=Z_{t, t-h}^{\top} Z_{t, t-h}+\int_{0}^{t} Z_{\tau, \tau-h}^{\top} Z_{\tau, \tau-h} \frac{d}{d t} r^{t-\tau} d \tau= \\
=Z_{t, t-h}^{\top} Z_{t, t-h}+\ln r \Gamma_{t}^{-1} \\
\dot{\Gamma}_{t}=-\Gamma_{t} Z_{t, t-h}^{\top} Z_{t, t-h} \Gamma_{t}-\ln r \Gamma_{t}
\end{gathered}
$$

that will conform the following estimation algorithm

$$
\begin{gathered}
\frac{d}{d t} \hat{C}_{t}=\Gamma_{t} Z_{\tau, \tau-h}^{\top}\left[F_{\tau, \tau-h}-Z_{t, t-h} \hat{C}_{t}\right] \\
\Gamma_{t}=-\Gamma_{t} Z_{t, t-h}^{\top} Z_{t, t-h} \Gamma_{t}-(\ln r) \Gamma_{t} \\
t \geq t_{0}:=\inf _{t}\left\{t \geq 0: \operatorname{det} \Gamma_{t}^{-1}=\operatorname{det}\left(\int_{0}^{t} Z_{\tau, \tau-h}^{\top} Z_{\tau, \tau-h} r^{t-\tau} d \tau\right)>0\right\} \\
\Gamma_{t_{0}}=\left[\int_{0}^{t} Z_{\tau, \tau-h}^{\top} Z_{\tau, \tau-h} r^{t-\tau} d \tau\right]^{-1}, \hat{C}_{t_{0}}=Y_{t_{0}} \Gamma_{t_{0}}
\end{gathered}
$$

\section{Estimation Error Analysis}

In this section the upper bound for the identification error of the 3 cases will be presented.

Theorem 1 (Additive white noise). Under assumptions A1-A5 the algorithm (3.19) provides the following upper bound for the estimation error

$$
\begin{gathered}
E\left\{\operatorname{tr}\left(\Delta A_{t}^{\top} \Gamma_{t}^{-2} \Delta A_{t}\right)\right\} \leq \\
\left(\sqrt[4]{X_{8}^{+} h} \delta_{A} \sqrt{\frac{1}{|2 \ln r|}\left[t^{2} r^{2 t}-\frac{t}{\ln r} r^{2 t}-\frac{1-r^{2 t}}{2(\ln r)^{2}}\right]}\right. \\
\left.+\left[\sqrt[8]{X_{8}^{+} h} \sqrt{\frac{D_{\sigma} h\left(1-r^{2 t}\right)}{|2 \ln r|}}+\sqrt[4]{\delta_{a}} \sqrt[8]{X_{8}^{+} h} \sqrt{\frac{1-r^{2 t}}{|2 \ln r|}}\right]\right)^{2}
\end{gathered}
$$

Proof. See Appendix. 
Theorem 2 (Colored noise). Under assumptions A1-A5 the algorithm (3.36) provides the following upper bound for $\Delta C_{t}=\hat{C}_{t}-C_{t}$

$$
\begin{aligned}
& E\left\{\operatorname{tr}\left(\Delta C_{t}^{\boldsymbol{\top}} \Gamma_{t}^{-2} \Delta C_{t}\right)\right\} \leq F_{t}\left(\delta_{A}, h\right):=\left(\sqrt{\delta_{P 1, t}}+\right. \\
& \left.\sqrt{\frac{\left(1-r^{2 t}\right)}{|2 \ln r|}}\left[\sqrt[8]{\delta^{2} L_{Z} h}+\sqrt[8]{L_{Z} h} \sqrt{D_{\sigma} D_{B} h}+\sqrt[8]{S_{8}^{+} L_{Z} h} \sqrt[4]{h D_{B}^{2}}\left(\Delta^{+}\right)\right]\right)^{2} \\
& h_{+}:=\max _{i}\left|h_{i}\right| \\
& L_{z}:=128\left(n^{8} L^{8} X_{8}^{+}+\left\|B_{t} H_{0}\right\|^{8}\right) \\
& \delta_{P 1, t}:=4 \sqrt{2 L_{z} h}\left(\frac{\delta_{A}^{2}}{|2 \ln r|}\left[t^{2} r^{2 t}-\frac{t}{\ln r} r^{2 t}-\frac{1-r^{2 t}}{2(\ln r)^{2}}\right]+\delta_{P 2, t}\right) \\
& \delta_{P 2, t}:=\frac{D_{\sigma} n}{2 h_{+}}\left[\frac{1-r^{2 t}}{|2 \ln r|}-\frac{1-\exp \left(2 t\left(h_{+}-|\ln r|\right)\right)}{\left(2 h_{+}+|2 \ln r|\right)}\right] \\
& \delta:=\sqrt{32 L_{z}}\left(h^{8}+D_{\sigma}^{2} n^{2} \frac{\left(2 h_{+} h+\exp \left(-2 h_{+} h\right)-1\right)^{2}}{h_{+}^{4}}\right)
\end{aligned}
$$

where $\mathrm{n}$ is the number of times that the regressor appears in $z_{t}$, that depends on the number of rows of matrix $A_{t}, h_{i}<0$ are the eigenvalues of matrix $H_{0}$, and in both theorems $h$ is the time delay of the back integration in (3.2) and (3.27).

Proof. See [21]

For the white multiplicative noise, taking into account the properties of the Itô integral (see [22] and [23]), the upper bound is the same than in the additive white noise.

Corollary 1. (on the effect of the "degree of color") In the general bound (4.2) the value $h_{+}$may be treated as the "degree of color" (when $h_{+}=0$ we deal with the "white-noise" case). The asymptotic bound can be structured as

$$
\begin{gathered}
F_{\infty}\left(\delta_{A}, h\right)=\left(\sqrt{c_{2}+\frac{c_{3}}{h_{+}}-\frac{c_{4}}{h_{+}\left(h_{+}+c_{5}\right)}}+\right. \\
\left.\left[\sqrt[4]{c_{6}+\left[-c_{7} h_{+}^{-1}+\frac{\exp \left(c_{8} h_{+}\right)}{h_{+}{ }^{2}}-h_{+}^{-2}\right]^{2}} c_{1}+c_{0}\right]\right)^{2}
\end{gathered}
$$

which graphic (for $x:=h_{+}, y:=F_{\infty}$ and some selected constants) corresponding to the function

$$
y(x)=\left(\sqrt{0.1+\frac{0.01}{x+1}}+\left(\sqrt[4]{0.1+\frac{0.1}{x^{4}}[\exp (x)-1-x]^{2}}+0.1\right)\right)^{2}
$$

is depicted at figure 1 .

It evidently shows that a higher the "degree of color" implies a higher bound of error estimating.

\section{Numerical Examples}

In this section will be shown the performance of the estimation algorithm for the 3 cases, applied to the some numerical problems, in order to verify the effectiveness of the method. 


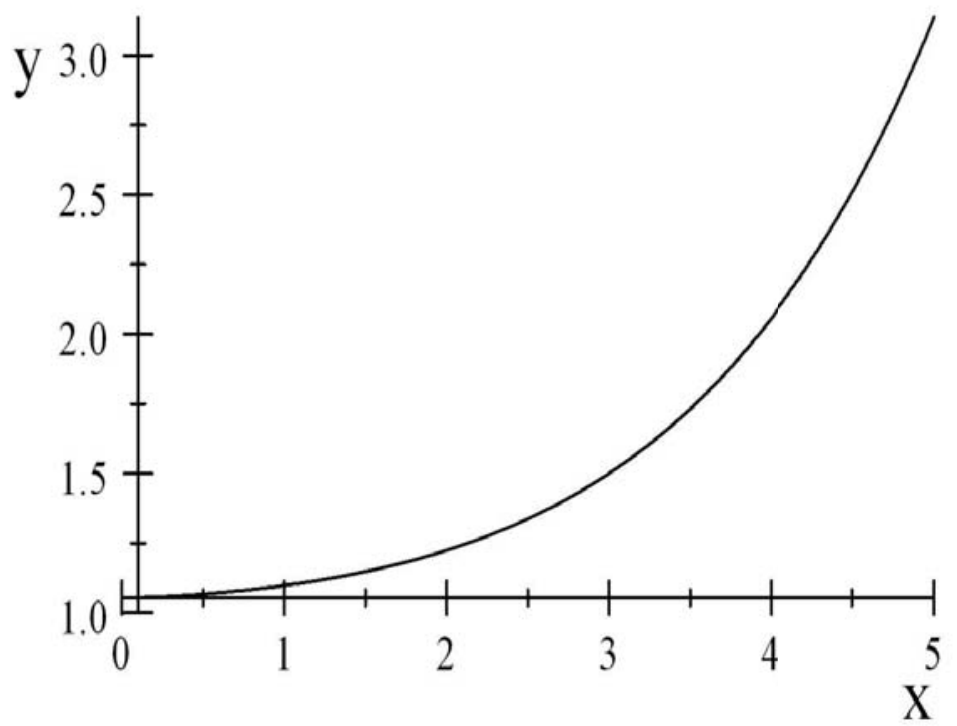

Figure 1. The dependence of the error estimation bound on the "color degree".

5.1. Example 1. We will estimate a single parameter, shown in figure 2. The excitation input is

$$
f_{t}=5 \sin (0.5 t)+12
$$

The sigma value is $\sigma_{t}=0.03$, for the colored noise we have $B_{t}=1, H_{t}=-3$, and $\Delta H_{t}=0.5 \sin (0.3 t)$. The initial condition and the time delay are $x_{t}=1.2$ and $h=0.02$. The forgetting factor, for the multiplicative and additive noise is $r=0.002$, and for the colored noise $r=0.1$.

Remark 4. The forgetting factor is adjusted by the "try-to-test" method. Our experience shows that for white noise this value should be small enough; while for the colored noise, since there are more uncertainties we cannot discard too much information. An optimal method to choose this factor it is already considered for further work.

5.1.1. Simulation results. The simulation results are shown in figures 2-4. In figure 2 one can see the performance of the method applied to a system under the 3 different kinds of perturbations. In figure 3, we can see the estimation under additive and multiplicative white noise, and in figure 4 it is shown a zoom of figure 3 in order to appreciate the differences in the estimation between an additive and a multiplicative noise.

The quality of the parameter estimation algorithm has been evaluated, for the 3 different kinds of noises, using the following performance index:

$$
J_{t}=\frac{1}{t+\varepsilon} \int_{0}^{t}\left(a_{i j, \tau}-\hat{a}_{i j, \tau}\right)^{2} d \tau, \quad \varepsilon=0.0001
$$


where $\varepsilon$ is a parameter that avoids a possible singularity. Based on the simulation results we obtained:

$$
\begin{gathered}
J_{t=16}=0.001398 \text { for additive noise } \\
J_{t=16}=0.001735 \text { for multiplicative noise } \\
J_{t=16}=1.691 \text { for colored noise }
\end{gathered}
$$

The figures clearly show the effect of the stochastic perturbations on the system. In figure 2 , and on the performance index, we can appreciate that the estimation algorithm has a better performance with the additive and multiplicative white noise, than with the colored noise, and in figure 4 we can appreciate the small differences of the algorithm between the additive and multiplicative noise.

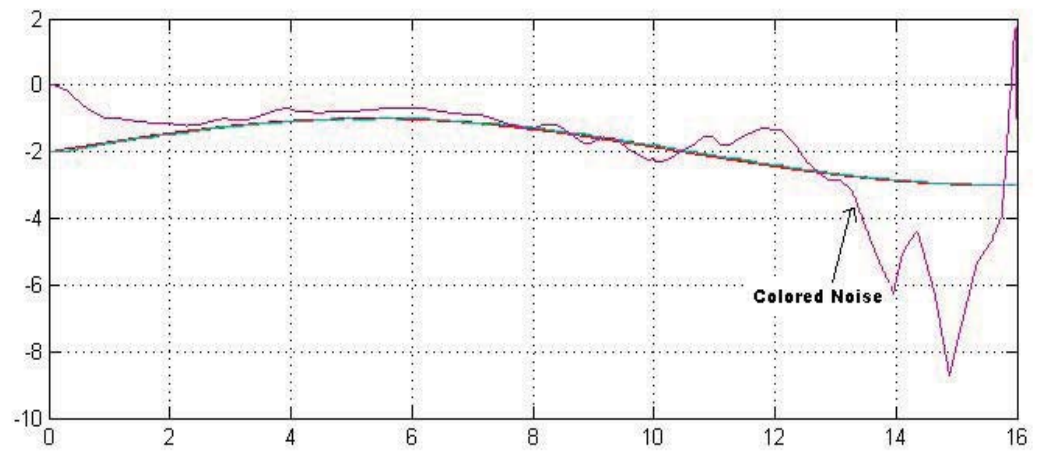

Figure 2. Parameter $a_{t}$ and its estimated.

5.2. Example 2. Here will be estimated the matrix $A_{t}$ as it is shown in Fig 5 . The excitation inputs are

$$
f_{1, t}=10 \sin (0.5 t)+12 ; f_{2, t}=10 \sin (0.2 t)+5
$$

The sigma values are $\sigma_{1}=\sigma 2=0.1$. The time delays for the multiplicative and additive white noise are $h_{1}=0.0008, h_{2}=0.001$, and the forgetting factor is $r=0.05$.

5.2.1. Simulation results. The results depicted in Fig. 5 show that there is not a visible difference in the performance of the algorithm for additive and multiplicative white noise.

For the colored noise case, some numerical results in time-varying matrix parameter estimation are shown in [21].

The performance index for this example shows the following results for additive white noise

$$
J_{t=50}^{(1,1)}=0.3446, J_{t=50}^{(1,2)}=0.006921, J_{t=50}^{(2,1)}=0.0079, J_{t=50}^{(2,2)}=1.313
$$

and for multiplicative white noise

$$
J_{t=50}^{(1,1)}=0.3493, J_{t=50}^{(1,2)}=0.007035, J_{t=50}^{(2,1)}=0.00798, J_{t=50}^{(2,2)}=1.325
$$

It is possible to see that even in the performance index there is just a minimum difference. 
As we can see in the first example, the forgetting factor for the multiplicative and additive noises is smaller compared to the colored noise, this is because the colored noise is formed by a forming filter with a deterministic and a stochastic part; and the other two perturbations are completely stochastic, another reason for this is that the forgetting factor discards information, and in the colored noise, since we have more uncertainties we cannot discard too much information, as previously mentioned, then the forgetting factor cannot take a very small value.

Another characteristic of this algorithm is that, for a colored noise, the simulation works just for a short period of time, while for additive and multiplicative white noise can work for a long period. From this point forward, it should be considered an alternative method for the system under a colored noise perturbation.

\section{Conclusion}

The LSM with forgetting factor is proposed for parameter estimation in stochastic systems. Here we suggest this method for 3 different kinds of perturbations, additive, multiplicative and colored noise. In this method it is no necessary to apply any kind of discretization, this implies a possible implementation in analogous and hybrid systems. The analysis of the error estimation is presented, and shows the dependence of the error on the degree of color. Some numerical examples show the quality in the proposed algorithm in a system under the 3 different kinds of perturbations.

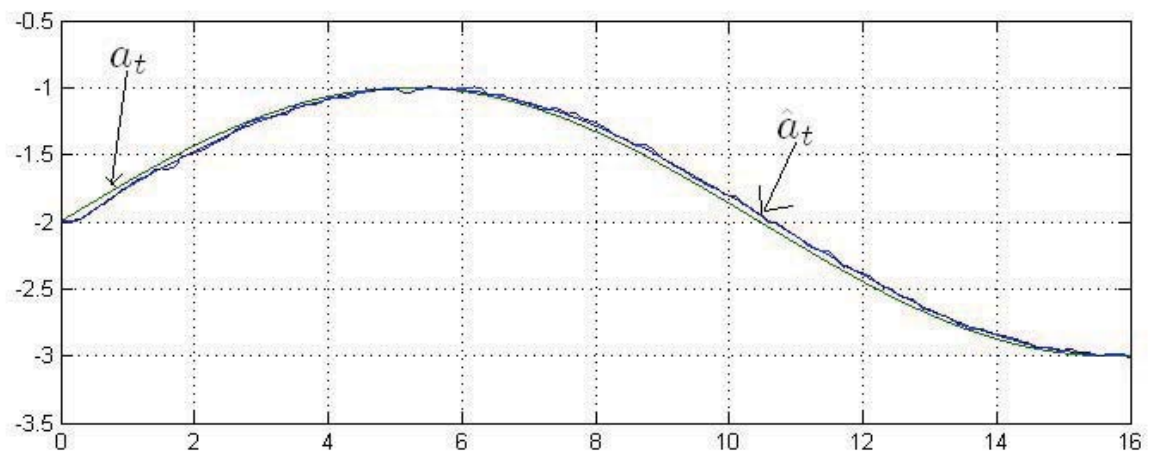

FIGURE 3. $a_{t}$ and its estimated under multiplicative and additive white noise.

\section{REFERENCES}

[1] Åström K., Introduction to Stochastic Control Theory, Academic Press, New York, San Francisco, London, 1970.

[2] Davis M.H.A., Linear Estimation and Stochastic Control, Chapman and Hall, London, 1977.

[3] Ljung L. "Perspectives on System Identification", Annual Reviews in Control, 34, pp. 1-12, 2010 .

[4] Ljung L., and Gunnarsson S., "Adaptation and Tracking in System Identification- A survey", Automatica, 26, pp. 7-21, 1990.

[5] Ljung L., "System Identification", , Proceedings of the Conf. on Signal Analysis and Prediction, ECSAP-97, Prague,pp 41-47, 1997. 
[6] Chen H. and Guo L., Identification and Stochastic Adaptive Control, Boston: Birkhäuser, 1991.

[7] Poznyak A., Escobar J., and Shtessel Y., "Sliding modes time varying matrix identification for stochastic systems", International Journal of System Sciences, 38, pp. 847-859, 2007.

[8] Chowdhury A., Rakshit B., and Saha P., "Parameter Estimation of a Delay Dynamical System using Synchronization in Presence of Noise", Chaos Solitons and Fractals, 32, pp. 1278-1284, 2007.

[9] Ljung L., System Identification: Theory for the User, Upper Saddle River, NJ: Prentice Hall, 1999.

[10] Kumar P., and Varaiya P., Stochastic Systems: Estimation, Identification and Adaptive Control, Englewood Cliffs, NJ:Prentice Hall, 1986

[11] Varziri M.S., McAuley K.B., and McLellan P.J.,"Parameter and state estimation in nonlinear stochastic continuous-time dynamic models with unknown disturbance intensity", Canadian Journal of Chemical Engineering, 86, 5, pp. 828-837, 2008.

[12] Varziri M.S., McAuley K.B., and McLellan P.J., "Parameter Estimation in Continuous-Time Dynamic Models in the Presence of Unmeasured States and Nonstationary Disturbances", Industrial \& Engineering Chemistry Research, 47, 2, pp. 380-393, 2008.

[13] Fan H., Söderström T., and Zou Y., "Continuous-Time AR Process Parameter Estimation in Presence of Additive White Noise", IEEE Transactions on Signal Processing, 47, 12, pp. 3392-3398, 1999.

[14] Favetto B., and Samson A., "Parameter Estimation for a Bidimentional Partially Observed Ornstein-Uhlenbeck Process with Biological Application"., Scandinavian Journal of Statistics, 37, pp 200-220, 2010.

[15] Yang Z., Sagara., and Wada K., "Identification of Continuous-time System from Sampled Input-Output Data Using Bias Eliminating Techniques", Control Theory and Advanced Technology, 9, pp. 53-57, 1993.

[16] Qinghua Zhang; Clavel, A., "Adaptive observer with exponential forgetting factor for linear time varying system", Proceedings of the 40th IEEE Conference on Decision and Control, 4,pp. 3886-3891,2001.

[17] GuoL., Ljung L., and Priouret P., "Performance analysis of the forgetting factor RLS algorithm", International Journal of Adaptive Control and Signal Processing, 7,6, pp. 525-537, 1993.

[18] Ding F., Chen T., "Performance bounds of forgetting factor least-squares algorithms for timevarying systems with finite measurement data", IEEE Transactions on Circuits and Systems I, 52,3,pp.555-566, 2005.

[19] Moore J.B., "Convergence of continuous time stochastic ELS parameter estimation", Stochastic processes and their applications, 1987,27, 195-215.

[20] Söderstrom T., Spectral Decomposition with Application to Identification, Numerical Techniques for Stochastic Systems, North Holland, Amsterdam.

[21] Escobar J., and Poznyak A., "Time-varying matrix estimation in stochastic continuous-time models under colored noise using LSM with forgetting factor". International Journal of System Science, 2010.

[22] Gard t., Introduction to Stochastic Differential Equations, Mercel Dekker New York, 1988.

[23] Poznyak A., Advanced Mathematical Tools for Automatic Control Engineers: Volume 2, Elsevier, 2009.

[24] Oksendal B., Stochastic Differential Equations. An Introduction with Applications. 5th Edition, Springer, 1998.

[25] Milshtein G.N., and Tret'yakov M.V., "Numerical Solution of Differential Equations with Colored Noise", Journal of Statistical Physics, 77, 3-4, pp. 691-715, 1994.

\section{Appendix}

Proof of Theorem 1. Define first

$$
\begin{aligned}
\Delta A_{t} & =\hat{A}_{t}-A_{t} \\
\Delta A_{t} & =Y_{t} \Gamma_{t}-A_{t}
\end{aligned}
$$




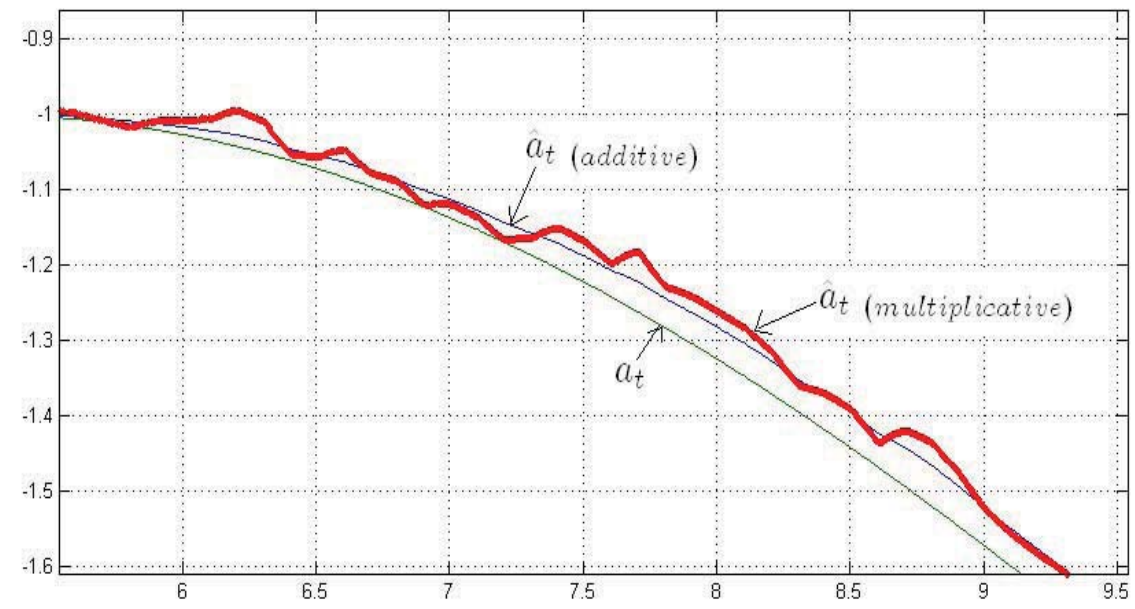

Figure $4 . a_{t}$ and its estimated under multiplicative and additive white noise.

replacing $Y_{t}$ we get

$$
\Delta A_{t}=\left(\int_{0}^{t} F_{\tau, \tau-h} X_{\tau, \tau-h}^{\top} r^{t-\tau} d \tau\right) \Gamma_{t}-A_{t}
$$

now we replace (3.3) in (7.1)

$$
\begin{gathered}
\Delta A_{t}=\left(\int_{0}^{t}\left(A_{\tau} X_{\tau, \tau-h}+\xi_{\tau, \tau-h}\right) X_{\tau, \tau-h}^{\top} r^{t-\tau} d \tau\right) \Gamma_{t}-A_{t} \\
=\left(\int_{0}^{t} A_{\tau} X_{\tau, \tau-h} X_{\tau, \tau-h}^{\top} r^{t-\tau} d \tau\right) \Gamma_{t}+\left(\int_{0}^{t}\left(\int_{-h}^{\tau} \sigma_{s} d W_{s}\right) X_{\tau, \tau-h}^{\top} r^{t-\tau} d \tau\right) \Gamma_{t} \\
+\left(\int_{0}^{t}\left(\int_{-h}^{\tau}\left(A_{s^{\prime}}-A_{\tau}\right) x_{s^{\prime}} d s^{\prime}\right) X_{\tau, \tau-h}^{\top} r^{t-\tau} d \tau\right) \Gamma_{t}-A_{t} \\
=\left(\int_{0}^{t}\left(A_{\tau}-A_{t}\right) X_{\tau, \tau-h} X_{\tau, \tau-h}^{\top} r^{t-\tau} d \tau\right) \Gamma_{t}+\left(\int_{0}^{t}\left(\int_{\sigma_{-h}}^{\tau} \sigma_{s} d W_{s}\right) X_{\tau, \tau-h}^{\top} r^{t-\tau} d \tau\right) \Gamma_{t} \\
+\left(\int_{0}^{t}\left(\int_{\sigma-h}^{\tau}\left(A_{s^{\prime}-A_{\tau}}\right) x_{s^{\prime}} d s^{\prime}\right) X_{\tau, \tau-h}^{\top} r^{t-\tau} d \tau\right) \Gamma_{t}
\end{gathered}
$$

or in equivalent form

$$
\begin{aligned}
\Gamma_{t}^{-1} \Delta A_{t} & =P_{1}+P_{3}^{\prime} \\
P_{3}^{\prime} & =P_{2}+P_{3}
\end{aligned}
$$



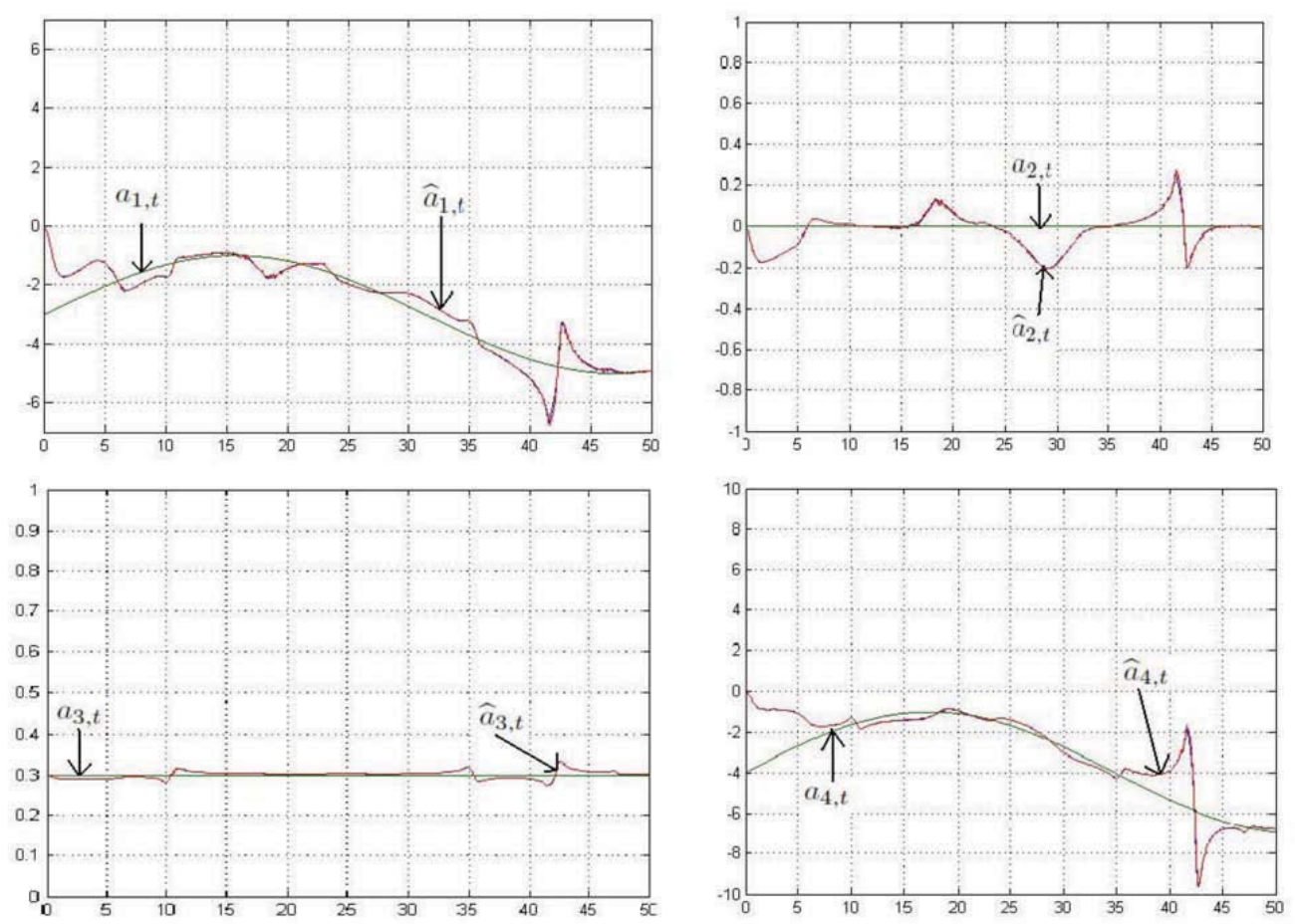

Figure 5. $A_{t}$ and its estimated under multiplicative and additive white noise.

$$
\begin{gathered}
P_{1}=-\int_{0}^{t}\left(A_{t}-A_{\tau}\right) X_{\tau, \tau-h} X_{\tau, \tau-h}^{\top} r^{t-\tau} d \tau \\
P_{2}=\int_{0}^{t}\left(\int_{-h}^{\tau} \sigma_{s} d W_{s}\right) X_{\tau, \tau-h}^{\top} r^{t-\tau} d \tau \\
P_{3}=\int_{0}^{t}\left(\int_{-h}^{\tau}\left(A_{s^{\prime}}-A_{\tau}\right) x_{s^{\prime}} d s^{\prime}\right) X_{\tau, \tau-h}^{\top} r^{t-\tau} d \tau
\end{gathered}
$$

in order to get the estimation error it's applied the next inequality

$$
E\left\{\operatorname{tr}\left(\Delta A_{t}^{\top} \Gamma_{t}^{-2} \Delta A_{t}\right)\right\} \leq(1+\lambda) E\left\{\operatorname{tr}\left(P_{1} P_{1}^{\top}\right)\right\}+\left(1+\lambda^{-1}\right) E\left\{\operatorname{tr}\left(P_{3^{\prime}} P_{3^{\prime}}^{\boldsymbol{\top}}\right)\right\}
$$

where

$$
\begin{gathered}
E\left\{\operatorname{tr}\left(P_{3^{\prime}} P_{3^{\prime}}^{\boldsymbol{\top}}\right)\right\} \leq\left(1+\lambda^{\prime}\right) E\left\{\operatorname{tr}\left(P_{2} P_{2}^{\boldsymbol{\top}}\right)\right\}+\left(1+\left(\lambda^{\prime}\right)^{-1}\right) E\left\{\operatorname{tr}\left(P_{3} P_{3}^{\boldsymbol{\top}}\right)\right\} \\
E\left\{\operatorname{tr}\left(P_{1} P_{1}^{\boldsymbol{\top}}\right)\right\}= \\
\int_{0}^{t} \int_{0}^{t} E \operatorname{tr}\left\{\left[\left(A_{t^{-}} A_{\tau}\right)\right] X_{\tau, \tau-h}^{\boldsymbol{\top}} X_{\tau, \tau-h}\left(A_{t^{-}} A_{\tau^{\prime}}\right)^{\boldsymbol{\top}} X_{\tau^{\prime}, \tau^{\prime}-h}^{\boldsymbol{\top}} X_{\tau^{\prime}, \tau^{\prime}-h} r^{t-\tau} r^{t-\tau^{\prime}} d \tau d \tau^{\prime}\right\}
\end{gathered}
$$




$$
\begin{gathered}
\left.\leq \int_{0}^{t} \int_{0}^{t} \sqrt[4]{E\left\{\left\|A_{t^{-}} A_{\tau}\right\|^{4} E\left\{\left\|A_{t^{-}} A_{\tau^{\prime}}\right\|^{4}\right\}\right.}\right\} \\
\sqrt[4]{E\left\{\left\|X_{\tau, \tau-h}^{\top} Z_{\tau, \tau-h}\right\|^{4}\right\} E\left\{\left\|X_{\tau^{\prime}, \tau^{\prime}-h}^{\top} X_{\tau^{\prime}, \tau^{\prime}-h}\right\|^{4}\right\}} r^{t-\tau} r^{t-\tau^{\prime}} d \tau d \tau^{\prime} \leq \\
{\left[\int_{0}^{t} \sqrt[4]{E\left\{\left\|A_{t}-A_{\tau}\right\|^{4}\right\}} \sqrt[4]{E\left\{\left\|X_{\tau, \tau-h}^{\top} X_{\tau, \tau-h}\right\|^{4}\right\}} r^{t-\tau} d \tau\right]^{2}}
\end{gathered}
$$

taking into account that $\left(A_{t}-A_{\tau}\right)=\int_{x=\tau}^{t} \dot{A}_{x} d x$, we get

$$
\left\|A_{t}-A_{\tau}\right\| \leq \delta_{A}(t-\tau)
$$

this yields

now for the term

$$
E\left\{\left\|A_{t^{-}}-A_{\tau}\right\|^{4}\right\} \leq \delta_{A}^{4}(t-\tau)^{4}
$$

$$
\begin{gathered}
E\left\{\left\|X_{\tau, \tau-h}^{\top} X_{\tau, \tau-h}\right\|^{4}\right\} \leq E\left\{\left\|X_{\tau, \tau-h}\right\|^{8}\right\} \\
=E\left\{\left\|\int_{s^{\prime}=t-h}^{t} x_{s^{\prime}} d s^{\prime}\right\|^{8}\right\} \leq E\left\{\int_{s^{\prime}=t-h}^{t}\left\|x_{s^{\prime}}\right\|^{8} d s^{\prime}\right\} \\
=\int_{s^{\prime}=t-h}^{t} E\left\{\left\|x_{s^{\prime}}\right\|^{8}\right\} d s^{\prime}
\end{gathered}
$$

considering A2

$$
\begin{gathered}
E\left\{\left\|x_{s^{\prime}}\right\|^{8}\right\} \leq X_{8}^{+} \\
E\left\{\left\|X_{\tau, \tau-h}\right\|^{8}\right\} \leq \int_{s^{\prime}=t-h}^{t} X_{8}^{+} d s^{\prime} \\
\leq X_{8}^{+} h
\end{gathered}
$$

then

$$
\begin{aligned}
E\left\{\operatorname{tr}\left(P_{1} P_{1}^{\top}\right)\right\} & \leq\left[\int_{0}^{t} \sqrt[4]{\delta_{A}^{4}(t-\tau)} \sqrt[4]{X_{8}^{+} h} r^{t-\tau} d \tau\right]^{2} \\
& =\sqrt{X_{8}^{+} h}\left[\int_{0}^{t} \delta_{A}(t-\tau) r^{t-\tau} d \tau\right]^{2} \\
& \leq \delta_{A}^{2} \sqrt{X_{8}^{+} h} \int_{0}^{t} x^{2} r^{2 x} d x
\end{aligned}
$$

since $\int_{0}^{t} x^{2} r^{2 x} d x=\frac{1}{2 \ln r}\left[t^{2} r^{2 t}-\frac{t}{\ln r} r^{2 t}-\frac{1-r^{2 t}}{2(\ln r)^{2}}\right]$ 
we get

$$
E\left\{\operatorname{tr}\left(P_{1} P_{1}^{\mathbf{\top}}\right)\right\} \leq \sqrt{X_{8}^{+} h} \frac{\delta_{A}^{2}}{2 \ln r}\left[t^{2} r^{2 t}-\frac{t}{\ln r} r^{2 t}-\frac{1-r^{2 t}}{2(\ln r)^{2}}\right]
$$

The term $E\left\{\operatorname{tr}\left(P_{2} P_{2}^{\mathbf{T}}\right)\right\}$ can be estimated in a similar way

$$
\begin{aligned}
& E\left\{\operatorname{tr}\left(P_{2} P_{2}^{\top}\right)\right\} \leq \\
& \int_{0}^{t} \int_{0}^{t} E\left\{t r\left(\int_{-h \tau-h}^{\tau} \int_{s^{\prime}}^{\tau} \sigma_{s^{\prime}} X_{\tau, \tau-h}^{\top} X_{\tau, \tau-h} d W_{s}^{\top} \sigma_{s}^{\top}\right)\right\} r^{2 t-\tau-\tau^{\prime}} d \tau d \tau^{\prime} \leq \\
& \int_{0}^{t} \int_{0}^{t} E\left\{\left\|X_{\tau, \tau-h}^{\top}\right\|^{2}\left\|\int_{\tau-h}^{\tau} \sigma_{s^{\prime}} d W_{s^{\prime}}\right\|^{2}\right\} r^{2 t-\tau-\tau^{\prime}} d \tau d \tau^{\prime} \leq \\
& \int_{0}^{t} \int_{0}^{t} \sqrt{E\left\{\left\|X_{\tau, \tau-h}^{\top}\right\|^{4}\right\}} \sqrt{E\left\{\left\|\int_{\tau-h}^{\tau} \sigma_{s^{\prime}} d W_{s^{\prime}}\right\|^{4}\right\}} r^{2 t-\tau-\tau^{\prime}} d \tau d \tau^{\prime}
\end{aligned}
$$
way

$$
\begin{gathered}
E\left\{\left\|\int_{\tau-h}^{\tau} \sigma_{s} d W_{s}\right\|^{4}\right\} \leq \\
E\left\{\int_{\tau-h \tau-h \tau-h \tau-h}^{\tau} \int_{\tau-h}^{\tau}\left\|\sigma_{s} \sigma_{s^{\prime}}^{\top}\right\|\left\|\sigma_{s^{\prime \prime}} \sigma_{s^{\prime \prime \prime}}^{\top}\right\| d W_{s} d W_{s^{\prime}}^{\top} d W_{s^{\prime \prime}} d W_{s^{\prime \prime \prime}}^{\top}\right\}= \\
\left(\int_{\sigma-h}^{\tau}\left\|\sigma_{s} \sigma_{s}^{\top}\right\| d s\right)^{2} \leq\left(\int_{\sigma-h}^{\tau} \operatorname{tr}\left\{\sigma_{s} \sigma_{s}^{\top}\right\} d s\right)^{2} \leq \\
\left(\int_{\sigma}^{\tau} d s\right)^{2}=D_{\sigma}^{2} h^{2}
\end{gathered}
$$

considering that $E\left\{\left\|X_{\tau, \tau-h}\right\|^{4}\right\} \leq E\left\{\left\|X_{\tau, \tau-h}\right\|^{8}\right\}^{1 / 2} \leq \sqrt{X_{8}^{+} h}, E\left\{\operatorname{tr}\left(P_{2} P_{2}^{\top}\right)\right\}$ can be estimated as

$$
\begin{aligned}
E\left\{\operatorname{tr}\left(P_{2} P_{2}^{\top}\right)\right\} & \leq \sqrt[4]{X_{8}^{+} h} \sqrt{D_{\sigma}^{2} h^{2}} \int_{0}^{t} \int_{0}^{t} r^{2 t-\tau-\tau^{\prime}} d \tau d \tau^{\prime} \\
& =\sqrt[4]{X_{8}^{+} h} D_{\sigma} h \frac{\left(1-r^{2 t}\right)}{|2 \ln r|}
\end{aligned}
$$


The term $E\left\{\operatorname{tr}\left(P_{3} P_{3}^{\boldsymbol{\top}}\right)\right\}$ is estimated in the following way

$$
\begin{gathered}
\leq E\left\{\left[\int_{0}^{t}\left(\int_{\sigma_{-h}^{\tau}}^{\tau}\left\|\left(A_{s^{\prime}}-A_{\tau}\right) x_{s^{\prime}}\right\| d s^{\prime}\right)\left\|X_{\tau, \tau-h}^{\top}\right\| r^{t-\tau} d \tau\right]^{2}\right\} \\
=E\left\{\left(\int_{0}^{t} \phi_{\tau}\left\|X_{\tau, \tau-h}^{\top}\right\| r^{t-\tau} d \tau\right)^{2}\right\}
\end{gathered}
$$

where

$$
\phi_{\tau}:=\int_{\tau-h}^{\tau}\left\|\left(A_{s^{\prime}}-A_{\tau}\right) x_{s^{\prime}}\right\| d s^{\prime}
$$

this yields to the following inequality

$$
E\left\{\operatorname{tr}\left(P_{3} P_{3}^{\top}\right)\right\} \leq \int_{0}^{t} \int_{0}^{t} \sqrt{E\left\{\phi_{\tau}^{2}\left\|X_{\tau, \tau-h}^{\top}\right\|^{2}\right\}} \sqrt{E\left\{\phi_{\tau^{\prime}}^{2}\left\|X_{\tau^{\prime}, \tau^{\prime}-h}^{\top}\right\|^{2}\right\}} r^{t-\tau} r^{t-\tau^{\prime}} d \tau d \tau^{\prime}
$$

the mathematical expectation $\sqrt{E\left\{\phi_{\tau}^{2}\left\|X_{\tau, \tau-h}^{\top}\right\|^{2}\right\}}$ can be rewritten as follows

$$
E\left\{\phi_{\tau}^{2}\left\|X_{\tau, \tau-h}^{\top}\right\|^{2}\right\} \leq \sqrt{E\left\{\phi_{\tau}^{4}\right\}} \sqrt{E\left\{\left\|X_{\tau, \tau-h}^{\top}\right\|^{4}\right\}}
$$

as in the previous estimations, we can conclude that

$$
E\left\{\phi_{\tau}^{4}\right\}=E\left\{\left(\int_{-h}^{\tau}\left\|\left(A_{s^{\prime}}-A_{\tau}\right) x_{s^{\prime}}\right\| d s^{\prime}\right)^{4}\right\} \leq
$$

$$
\begin{gathered}
\int_{\tau-h \tau-h \tau-h \tau-h}^{\tau} \int_{\tau}^{\tau} \int_{-h}^{\tau}\left(E\left\{\left\|x_{s}\right\|^{8}\right\} E\left\{\left\|\left(A_{s^{-}} A_{\tau}\right)\right\|^{8}\right\} E\left\{\left\|x_{s^{\prime}}\right\|^{8}\right\} E\left\{\left\|\left(A_{s^{\prime}}-A_{\tau}\right)\right\|^{8}\right\}\right. \\
\left.E\left\{\left\|x_{s^{\prime \prime}}\right\|^{8}\right\} E\left\{\left\|\left(A_{s^{\prime \prime}}-A_{\tau}\right)\right\|^{8}\right\} E\left\{\left\|x_{s^{\prime \prime}}\right\|^{8}\right\} E\left\{\left\|\left(A_{s^{\prime \prime \prime}}-A_{\tau}\right)\right\|^{8}\right\}\right)^{1 / 8} d s d s^{\prime} d s^{\prime \prime} d s^{\prime \prime \prime} \\
=\left[\int_{\tau-h}^{\tau}\left(E\left\{\left\|x_{s^{\prime}}\right\|^{8}\right\} E\left\{\left\|\left(A_{s^{\prime}}-A_{\tau}\right)\right\|^{8}\right\}\right)^{1 / 8} d s^{\prime}\right]^{4}
\end{gathered}
$$

from the equation (7.2) we can estimate $A_{\mathcal{s}^{\prime}}-A_{\tau}$

$$
E\left\{\left\|\left(A_{s^{\prime}}-A_{\tau}\right)\right\|^{8}\right\} \leq \delta_{A}^{8}\left(s^{\prime}-\tau\right)^{8}
$$

and for $E\left\{\left\|x_{s^{\prime}}\right\|^{8}\right\}$ we get

$$
E\left\{\left\|x_{s^{\prime}}\right\|^{8}\right\} \leq X_{8}^{+}
$$


from this point, we can rewrite (7.3) as follows

$$
\begin{aligned}
\delta_{a}:= & {\left[\int_{\tau-h}^{\tau}\left(E\left\{\left\|x_{s^{\prime}}\right\|^{8}\right\} E\left\{\left\|\left(A_{s^{\prime}}-A_{\tau}\right)\right\|^{8}\right\}\right)^{1 / 8} d s^{\prime}\right]^{4} } \\
& \leq \sqrt{X_{8}^{+}}\left(\int_{-h}^{\tau} \delta_{A}\left(s^{\prime}-\tau\right) d s^{\prime}\right)=\sqrt[8]{X_{8}^{+}} \frac{h^{8}}{16}
\end{aligned}
$$

taking into account

$$
\begin{aligned}
\int_{0}^{t} \int_{0}^{t} \sqrt[4]{E\left\{\phi_{\tau}^{4}\right\}} \sqrt[4]{E\left\{\left\|X_{\tau, \tau-h}^{\top}\right\|^{4}\right\}} \sqrt[4]{E\left\{\phi_{\tau^{\prime}}^{4}\right\}} \sqrt[4]{E\left\{\left\|X_{\tau^{\prime}, \tau^{\prime}-h}\right\|^{4}\right\}} r^{t-\tau} r^{t-\tau^{\prime}} d \tau d \tau^{\prime} \\
=\left[\int_{0}^{t} \sqrt[4]{E\left\{\phi_{\tau}^{4}\right\}} \sqrt[4]{E\left\{\left\|X_{\tau, \tau-h}^{\top}\right\|^{4}\right\}} r^{t-\tau} d \tau\right]^{2} \\
\leq\left[\sqrt[4]{\delta_{a}} \int_{0}^{t} \sqrt[4]{E\left\{\left\|X_{\tau, \tau-h}^{\top}\right\|^{4}\right\}} r^{t-\tau} d \tau\right]^{2^{t}}
\end{aligned}
$$

and using Lyapunov inequality

$$
E\left\{\left\|X_{\tau, \tau-h}^{\top}\right\|^{4}\right\} \leq \sqrt{X_{8}^{+} h}
$$

we get

$$
\begin{gathered}
{\left[\sqrt[4]{\delta_{a}} \int_{0}^{t} \sqrt[4]{E\left\{\left\|X_{\tau, \tau-h}^{\top}\right\|^{4}\right\}} r^{t-\tau} d \tau\right]^{2}} \\
\leq \sqrt{\delta_{a}} \sqrt[4]{X_{8}^{+} h} \int_{0}^{t} r^{2(t-\tau)} d \tau
\end{gathered}
$$

then the term $E\left\{\operatorname{tr}\left(P_{3} P_{3}^{\top}\right)\right\}$ can be estimated as follows

$$
E\left\{\operatorname{tr}\left(P_{3} P_{3}^{\boldsymbol{\top}}\right)\right\} \leq \frac{\sqrt{\delta_{a}} \sqrt[4]{X_{8}^{+} h}}{|2 \ln r|} 1-r^{2 t}
$$

now we replace all the terms in

$$
\begin{gathered}
E\left\{\operatorname{tr}\left(\Delta A_{t}^{\top} \Gamma_{t}^{-2} \Delta A_{t}\right)\right\} \leq(1+\lambda) E\left\{\operatorname{tr}\left(P_{1} P_{1}^{\boldsymbol{\top}}\right)\right\} \\
+\left(1+\lambda^{-1}\right) E\left\{\left(1+\lambda^{\prime}\right) E\left\{\operatorname{tr}\left(P_{2} P_{2}^{\boldsymbol{\top}}\right)\right\}+\left(1+\left(\lambda^{\prime}\right)^{-1}\right) E\left\{\operatorname{tr}\left(P_{3} P_{3}^{\boldsymbol{\top}}\right)\right\}\right\}
\end{gathered}
$$

and using the next inequality

$$
(1+\lambda) a+\left(1+\lambda^{-1}\right) b \geq(\sqrt{a}+\sqrt{b})^{2}, a, b>0
$$


where $\lambda=\sqrt{b / a}$, we get

$$
\begin{gathered}
E\left\{\operatorname{tr}\left(\Delta A_{t}^{\top} \Gamma_{t}^{-2} \Delta A_{t}\right)\right\} \leq(1+\lambda)\left\{\sqrt{X_{8}^{+} h} \frac{\delta_{A}^{2}}{2 \ln r}\left[t^{2} r^{2 t}-\frac{t}{\ln r} r^{2 t}-\frac{1-r^{2 t}}{2(\ln r)^{2}}\right]\right\} \\
+\left(1+\lambda^{-1}\right)\left\{\left[\sqrt{\sqrt[4]{X_{8}^{+} h} D_{\sigma} h \frac{\left(1-r^{2 t}\right)}{|2 \ln r|}}+\sqrt{\frac{\sqrt{\delta_{a} \sqrt[4]{X_{8}^{+} h}}}{|2 \ln r|}-r^{2 t}}\right]^{2}\right\} \\
\leq\left(\sqrt[4]{X_{8}^{+} h} \delta_{A} \sqrt{\frac{1}{2 \ln r}\left[t^{2} r^{2 t}-\frac{t}{\ln r} r^{2 t}-\frac{1-r^{2 t}}{2(\ln r)^{2}}\right]}\right. \\
\left.+\left[\sqrt[8]{X_{8}^{+} h} \sqrt{\frac{D_{\sigma} h\left(1-r^{2 t}\right)}{|2 \ln r|}}+\sqrt[4]{\delta_{a}} \sqrt[8]{X_{8}^{+} h} \sqrt{\frac{1-r^{2 t}}{|2 \ln r|}}\right]\right)^{2}
\end{gathered}
$$

Division of Automatic Control,University of Linkping, 58183 LinkPing Sweden E-mail address: escobar@isy.liu.se 